\title{
Cellular Telephone
}

National Cancer Institute

\section{Source}

National Cancer Institute. Cellular Telephone. NCI Thesaurus. Code C60787.

A hand-held mobile radiotelephone for use in an area divided into small sections (cells), each with its own short-range transmitter/receiver 